\title{
$\beta$-Hydroxy- $\beta$-methylbutyrate free acid reduces markers of exercise-induced muscle damage and improves recovery in resistance-trained men
}

\author{
Jacob M. Wilson ${ }^{1 *}$, Ryan P. Lowery ${ }^{1}$, Jordan M. Joy ${ }^{1}$, Joe A. Walters ${ }^{1}$, Shawn M. Baier ${ }^{2}$, \\ John C. Fuller $\mathrm{Jr}^{2}$, Jeffrey R. Stout ${ }^{3}$, Layne E. Norton ${ }^{1,4}$, Eric M. Sikorski ${ }^{5}$, Stephanie M. C. Wilson ${ }^{6}$, \\ Nevine M. Duncan ${ }^{1}$, Nelo E. Zanchi ${ }^{1}$ and John Rathmacher ${ }^{2,7}$ \\ ${ }^{1}$ Department of Health Sciences and Human Performance, University of Tampa, Tampa, FL 33606, USA \\ ${ }^{2}$ Metabolic Technologies, Inc., Iowa State University Research Park, Ames, IA, USA \\ ${ }^{3}$ Sport and Exercise Science, College of Education, University of Central Florida, Orlando, FL, USA \\ ${ }^{4}$ Biolayne, Inc., Tampa, FL, USA \\ ${ }^{5}$ Department of Biology, University of Tampa, Tampa, FL, USA \\ ${ }^{6}$ Department of Nutrition, IMG Performance Institute, IMG Academy, Bradenton, FL, USA \\ ${ }^{7}$ Department of Animal Science, Iowa State University, Ames, IA, USA
}

(Submitted 17 August 2012 - Final revision received 19 October 2012 - Accepted 29 October 2012 - First published online 3 January 2013)

\begin{abstract}
The purpose of the present study was to determine the effects of short-term supplementation with the free acid form of $\beta$-hydroxy$\beta$-methylbutyrate (HMB-FA) on indices of muscle damage, protein breakdown, recovery and hormone status following a high-volume resistance training session in trained athletes. A total of twenty resistance-trained males were recruited to participate in a high-volume resistance training session centred on full squats, bench presses and dead lifts. Subjects were randomly assigned to receive either $3 \mathrm{~g} / \mathrm{d}$ of $\mathrm{HMB}-\mathrm{FA}$ or a placebo. Immediately before the exercise session and $48 \mathrm{~h}$ post-exercise, serum creatine kinase (CK), urinary 3-methylhistadine (3-MH), testosterone, cortisol and perceived recovery status (PRS) scale measurements were taken. The results showed that CK increased to a greater extent in the placebo (329\%) than in the HMB-FA group (104\%) $(P=0 \cdot 004, d=1 \cdot 6)$. There was also a significant change for PRS, which

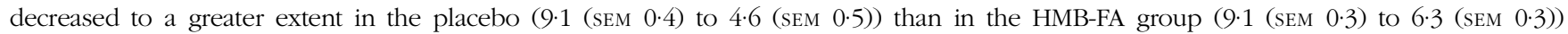
$(P=0.005, d=-0 \cdot 48)$. Muscle protein breakdown, measured by $3-\mathrm{MH}$ analysis, numerically decreased with HMB-FA supplementation and approached significance $(P=0 \cdot 08, d=0 \cdot 12)$. There were no acute changes in plasma total or free testosterone, cortisol or C-reactive protein. In conclusion, these results suggest that an HMB-FA supplement given to trained athletes before exercise can blunt increases in muscle damage and prevent declines in perceived readiness to train following a high-volume, muscle-damaging resistance-training session.
\end{abstract}

Key words: $\beta$-Hydroxy- $\beta$-methylbutyrate free acid: Muscle damage: Recovery: Hormones

Scientists have attempted to increase training-induced adaptations through a number of protocols, which generally aim at augmenting and/or speeding skeletal muscle regeneration $^{(1-3)}$. Effective training protocols for increasing lean body mass and strength often leave athletes in a fatigued state, which can limit overall training frequency ${ }^{(4)}$. Oral administration of the leucine metabolite $\beta$-hydroxy- $\beta$-methylbutyrate $(\mathrm{HMB})$ has been associated with increases in lean body mass ${ }^{(5,6)}$, strength $^{(7)}$ and power ${ }^{(8)}$. HMB is presently thought to work by speeding regenerative capacity as evidenced by increased protein synthesis and decreased muscle protein breakdown, damage and soreness following high-intensity exercise ${ }^{(7)}$. Recent data suggest that HMB acts through multiple mechanisms that include the simultaneous stimulation of the mammalian target of rapamycin, the major kinase directing translation initiation of muscle protein synthesis ${ }^{(9,10)}$, and the inhibition of the ubiquitin-proteasome ${ }^{(11,12)}$ pathway, the major regulatory system of protein degradation in skeletal muscle ${ }^{(13)}$.

The overwhelming majority of studies using HMB have examined its results after one or more weeks of training and supplementation $^{(5-7,14,15)}$. To date, only one acute HMB study has been conducted. In this study, Wilson et al. ${ }^{(16)}$ examined the acute effects of an oral Ca HMB (HMB-Ca)

Abbreviations: 3-MH, 3-methylhistadine; CK, creatine kinase; CRP, C-reactive protein; HMB, $\beta$-hydroxy- $\beta$-methylbutyrate; HMB-Ca, calcium $\beta$-hydroxy- $\beta$ methylbutyrate; HMB-FA, $\beta$-hydroxy- $\beta$-methylbutyrate free acid; PRS, perceived recovery status. 
supplement on sixteen untrained males using a unilateral, isokinetic leg extension-based training protocol. These researchers found that $\mathrm{HMB}-\mathrm{Ca}$ consumed before exercise blunted the rise in lactate dehydrogenase relative to the supplement consumed following exercise or a placebo. This study was conducted using an untrained population and controversy exists over whether HMB is effective in highly trained individuals ${ }^{(17)}$. In addition, the training stimulus used in that study was neither traditional nor practical for eliciting desirable body mass and strength changes in a trained population. Finally, HMB-Ca used by Wilson et al. ${ }^{(16,17)}$ has a slow rate of appearance, taking approximately 60-120 min to reach peak plasma values ${ }^{(18)}$. Due to the slow rate of appearance of HMB-Ca and the need for its ingestion much before training, HMB-Ca may be impractical for everyday use in an exercise regimen.

Recently, Fuller et $a l .{ }^{(18)}$ observed that an HMB free acid (HMB-FA) supplement was absorbed more rapidly, peaked faster (30 $\mathrm{min})$ and had a greater clearance to tissues than an HMB-Ca supplement, thus shortening the length of time needed between supplementation and exercise.

Research is lacking on HMB and resistance-trained athletes using a practical and high-intensity training stimulus. Therefore, the purpose of the present study was to investigate the acute effects of an HMB-FA supplement administered just before a bout of resistance exercise on serum indices of muscle damage, inflammation, perceived recovery, muscle protein breakdown, and anabolic and catabolic hormone status in resistance-trained men.

\section{Methods}

\section{Subjects}

A total of twenty resistance-trained males aged 21.6 (SEM 0.5) years with an average squat, bench press and dead lift of 1.7 (SEM 0.04), 1.3 (SEM 0.04) and 2.0 (SEM 0.05) times their body weight were recruited for the study (Table 1 ). Subjects could not participate if they were currently taking anti-inflammatory agents, any other performance-enhancing supplement or if they smoked. The present study was registered at ClinicalTrials.gov (registration

Table 1. Subject characteristics

(Mean values with their standard errors, $n$ 20)

\begin{tabular}{|c|c|c|c|c|}
\hline \multirow[b]{3}{*}{ Variables } & \multicolumn{4}{|c|}{ Treatments } \\
\hline & \multicolumn{2}{|c|}{ Placebo ( $n 9)$} & \multicolumn{2}{|c|}{$\mathrm{HMB}^{*}(n 11)$} \\
\hline & Mean & SEM & Mean & SEM \\
\hline Age (years) & 22 & 0.8 & 20 & $1 \cdot 7$ \\
\hline Height $(\mathrm{cm})$ & $180 \cdot 9$ & $2 \cdot 5$ & 178.9 & 1.9 \\
\hline Weight (kg) & $87 \cdot 1$ & $4 \cdot 8$ & $83 \cdot 1$ & $2 \cdot 8$ \\
\hline DEXA BF (\%) & $21 \cdot 2$ & 1.2 & $20 \cdot 4$ & 1.4 \\
\hline DEXA LBM $(\mathrm{kg})$ & $68 \cdot 3$ & $2 \cdot 9$ & $66 \cdot 2$ & $2 \cdot 6$ \\
\hline Total strength $(\mathrm{kg})$ & $422 \cdot 2$ & $30 \cdot 1$ & $430 \cdot 4$ & $20 \cdot 9$ \\
\hline Total volume lifted $(\mathrm{kg})$ & $17965 \cdot 2$ & 1742.9 & $17949 \cdot 1$ & $1145 \cdot 6$ \\
\hline
\end{tabular}

HMB, $\beta$-hydroxy- $\beta$-methylbutyrate; DEXA, dual-energy X-ray absorptiometry; BF, body fat; LBM, lean body mass.

* $3 \mathrm{~g} \mathrm{HMB}$ free acid/d in three $1 \mathrm{~g}$ doses. no. NCT01508338). The study was conducted according to the guidelines laid down in the Declaration of Helsinki, and the University of Tampa Institutional Review Board approved all procedures involving human subjects. Written informed consent was obtained from all subjects.

\section{Baseline strength and body composition testing}

At the start of the study, subjects reported to the laboratory for baseline one maximal repetition testing of the full squat, bench press and dead lift, and to determine their body composition on a GE Lunar Prodigy dual X-ray absorptiometry apparatus (software version, enCORE 2008). Each lift was deemed successful as described by the International Powerlifting Federation rules ${ }^{(19)}$. Total body strength was calculated as the sum of the squat, bench press and dead lift one maximal repetition values.

\section{Supplementation and resistance exercise protocol}

Before the exercise session, subjects were randomly assigned to receive either $3 \mathrm{~g} / \mathrm{d}$ of HMB-FA (combined with food-grade orange flavours and sweeteners) or a placebo (food-grade orange flavours and sweeteners) divided equally into three servings given $30 \mathrm{~min}$ before exercise and again with lunch and the evening meals. To ensure compliance, investigators watched as the subjects consumed the supplement before the exercise session. On the non-training days, subjects were instructed to consume one packet with three separate meals throughout the day. Empty packets were presented to the investigators upon returning to the laboratory following nontraining days. All subjects participated in a high-volume resistance training session consisting of three sets of full squats, bench press, dead lifts, pull-ups, barbell bent over rows, parallel dips, military press, barbell curls and triceps extensions. Each exercise was performed for three sets of twelve maximal repetitions intensity, with a supervised and timed rest period length of $1 \mathrm{~min}$ between the sets. Training volume for the exercise session was calculated as the product of total sets, repetitions and weight lifted during each exercise.

\section{Resting blood draws}

Resting blood draws were obtained via venepuncture after a $12 \mathrm{~h}$ fast by a trained phlebotomist immediately before training and at $48 \mathrm{~h}$ after the resistance training bout, as this is when we have found that creatine kinase (CK) peaks in the blood $^{(16)}$. Whole blood was collected and transferred into appropriate tubes for obtaining serum and plasma, and centrifuged at $1500 \mathrm{~g}$ for $15 \mathrm{~min}$ at $4^{\circ} \mathrm{C}$. Resulting serum and plasma were divided into aliquots and stored at $-80^{\circ} \mathrm{C}$ until subsequent analyses.

\section{Biochemical analysis}

Samples were thawed once and analysed in duplicate for each analyte. All blood draws were scheduled at the same time of day to negate confounding influences of diurnal hormonal 
variations. Serum total testosterone, cortisol and C-reactive protein (CRP) were assayed via ELISA kits obtained from Diagnostic Systems Laboratories. All hormones were measured in the same assay on the same day to avoid compounded inter-assay variance. Intra-assay variance was less than $3 \%$ for all analytes. Serum CK was measured using colorimetric procedures at $340 \mathrm{~nm}$ (Diagnostics Chemicals). Subjects were instructed to consume a meat-free diet $72 \mathrm{~h}$ before urine collection in order to prevent exogenous intake of 3-methylhistidine (3-MH). Urine collection occurred on two occasions. The first occurred the day before training. The second occurred $48 \mathrm{~h}$ following training. On both occasions, urine was collected for a period of $24 \mathrm{~h}$. A 3 -litre amber collection container and instructions were given to each subject before the urine collection period, and the subjects were instructed to start the collection after voiding their bladder in the morning and to continue for $24 \mathrm{~h}$ until voiding their urine the following morning. The subjects were instructed to keep the collection container refrigerated throughout the collection period. Urine volume was recorded, and a sample was taken and stored at $-80^{\circ} \mathrm{C}$. Urinary $3-\mathrm{MH}$ was determined from the $24 \mathrm{~h}$ urine collection by previously described methods (Metabolic Technologies) ${ }^{(20)}$. Urinary creatinine was measured using a colorimetric Jaffe reaction (Cayman Chemical). The 3-MH data were expressed as a 3-MH:creatinine ratio.

\section{Perceived recovery status scale}

The perceived recovery status (PRS) scale was taken before and $48 \mathrm{~h}$ following the training session. The PRS score consists of values between 0 and 10 , with $0-2$ being very poorly

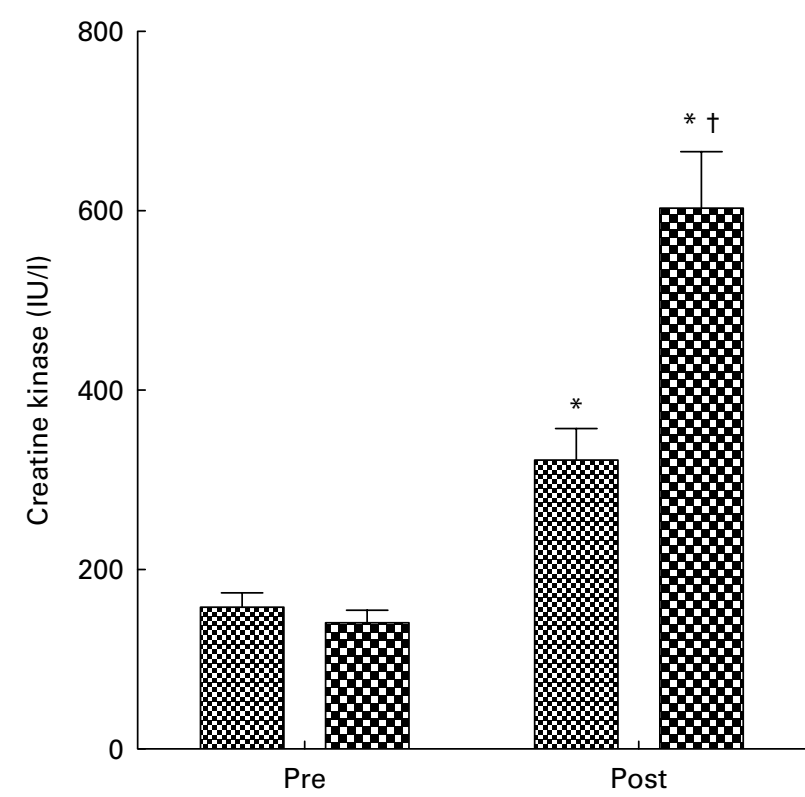

Fig. 1. Acute effects of $\beta$-hydroxy- $\beta$-methylbutyrate free acid (HMB-FA) on serum creatine kinase (CK). Resistance-trained males (n 20) were supplemented three times daily starting $30 \mathrm{~min}$ before an exercise bout with either HMB-FA (圆, $1 \mathrm{~g}$ ) or a placebo $(\mathbf{\nabla})$. HMB-FA significantly blunted the rise in $\mathrm{CK}$ (treatment effect $P<0.004$ ). ${ }^{*}$ Mean value was significantly different from baseline $(P<0.05)$. † Mean value was significantly different from the HMB-FA group $(P<0.05)$. recovered and with anticipated declines in performance, 4-6 being low to moderately recovered and expected similar performance and 8-10 representing high perceived recovery with expected increases in performance.

\section{Diet control}

Subjects could not have taken nutritional supplements for at least 3 months before data collection. At 2 weeks before and throughout the study, subjects were placed on a diet consisting of $25 \%$ protein, $50 \%$ carbohydrates and $25 \%$ fat, which was designed by a registered dietitian who specialised in sport nutrition (Registered Dietitian, Licensed Dietitian/ Nutritionist, and Certified Sports Nutritionist).

\section{Statistical analysis}

The treatment main effect was evaluated by an ANCOVA on all dependent variables. The mixed model in SAS (version 9.1; SAS, Inc.) was used to analyse the acute changes from baseline to $48 \mathrm{~h}$. The baseline values were used as a covariate. For effect size, the Cohen statistic was calculated, and according to Cohen ${ }^{(21)}, d$ values of $0 \cdot 2,0.5$ and 0.8 represent small, medium and large effect sizes, respectively. An $\alpha$ of $P<0.05$ was established a priori.

\section{Results}

\section{Subject characteristics and total training volume}

There were no differences between the groups for age, height, body weight, lean body mass or percentage of body fat (Table 1). The subjects studied were experienced in weight training and could be described as a muscular body type. There was no difference in total strength (sum of bench press, squat and dead lift one maximal repetition) between the groups, and total strength was 422 (SEM 30) and 430 (SEM 21) kg for placebo- and HMB-FA-supplemented groups, respectively. There was also no significant difference in total training volume (sets $\times$ repetitions $\times$ total weight lifted) completed for the exercise session between the groups (Table 1).

\section{Changes in serum creatine kinase, perceived recovery status scale, urinary 3-methylhistidine, testosterone, free testosterone, cortisol and C-reactive protein}

The high-volume exercise protocol resulted in a significant acute change in serum $\mathrm{CK}$, which increased to a greater extent in the placebo $(329 \%)$ than in the HMB-FA group (104\%) ( $P=0 \cdot 004, d=1 \cdot 6$; Fig. 1). Before the exercise session, serum CK levels were the same in placebo- and HMB-FA-supplemented subjects (141 (SEM 14) and 158 (SEM 16) IU/l, respectively). As a result of the exercise session, serum CK in the placebo group increased to 604 (SEM 83) IU/1 after $48 \mathrm{~h}$ while the HMB-FA-supplemented group showed a much diminished increase to only 322 (SEM 35) IU/1 $(P=0.004, \quad d=1.6)$. Supplementation with HMB-FA also resulted in an improved PRS score, an indication of a quicker 
recovery from the intense exercise session and potential for improved performance in subsequent sessions. The PRS score decreased from 9.1 (SEM 0.4) to 4.6 (SEM 0.5) $48 \mathrm{~h}$ after the exercise session in the placebo group, while in the HMB-FA group, the decrease was much less, decreasing from $9 \cdot 1$ (SEM 0.3$)$ to $6 \cdot 3$ (SEM 0.3 ) from pre- to post-exercise $(P=0.005, d=-0.48 ;$ Fig. 2$)$. The PRS score indicated that the HMB-FA-supplemented subjects had recovered to a greater extent $48 \mathrm{~h}$ post-exercise than the placebo-supplemented group. While not significantly different between the groups, muscle protein breakdown, as measured by urinary 3-MH:creatinine ratio, remained constant in the placebo-supplemented group and approached a statistically lower rate of muscle protein degradation in the HMB-FA-supplemented group $48 \mathrm{~h}$ post-exercise $(P=0 \cdot 08, d=0 \cdot 12$; Table 2$)$. There were no acute changes in plasma total or free testosterone, cortisol or CRP (Tables 2 and 3).

\section{Discussion}

The primary aim of the present study was to determine the acute effects of HMB-FA administration in resistance-trained men on changes in serum CK, perceived recovery, anabolic and catabolic hormone status, protein breakdown and inflammation. The major findings of the present study were that HMB-FA sped recovery, blunted the decrease in PRS and may lead to decreased muscle protein breakdown.

\section{Creatine kinase}

CK is a commonly used indicator of myofibre disruption, which peaks $48 \mathrm{~h}$ post-resistance training ${ }^{(16)}$. As such, CK

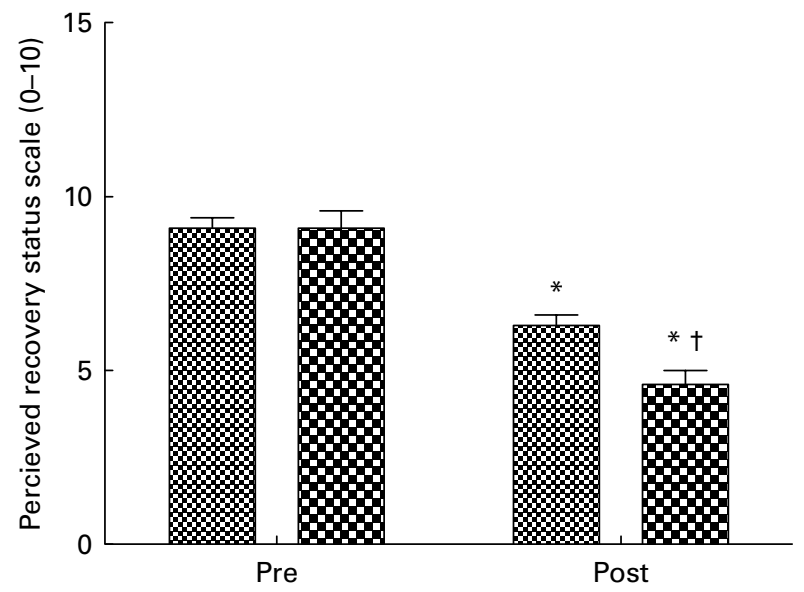

Fig. 2. Acute effects of $\beta$-hydroxy- $\beta$-methylbutyrate free acid (HMB-FA) on perceived recovery status (PRS) scale. Resistance-trained males ( $n$ 20) were supplemented three times daily starting $30 \mathrm{~min}$ before an exercise bout with either HMB-FA (圈, $1 \mathrm{~g}$ ) or a placebo $(\boldsymbol{g})$. The PRS score consists of values between 0 and 10 , with $0-2$ being very poorly recovered and with anticipated declines in performance, $4-6$ being low to moderately recovered and expected similar performance and 8-10 representing high perceived recovery with expected increases in performance. HMB-FA supplementation resulted in improved PRS after the exercise bout (treatment effect $P<0.005$ ). * Mean value was significantly different from baseline $(P<0.05)$. † Mean value was significantly different from the HMB-FA group $(P<0.05)$.
Table 2. Effects of $\beta$-hydroxy- $\beta$-methylbutyrate $(\mathrm{HMB})$ free acid on 3-methylhistadine and $\mathrm{C}$-reactive protein

(Mean values with their standard errors)

\begin{tabular}{|c|c|c|c|c|c|}
\hline & \multicolumn{4}{|c|}{ Time points } & \multirow[b]{3}{*}{$P^{*}$} \\
\hline & \multicolumn{2}{|c|}{0} & \multicolumn{2}{|c|}{$48 \mathrm{~h}$} & \\
\hline & Mean & SEM & Mean & SEM & \\
\hline \multicolumn{6}{|c|}{ 24h 3-MH:CR ( $\mu \mathrm{mol}: \mathrm{mg})$} \\
\hline Placebo & 0.131 & 0.006 & 0.131 & 0.004 & \\
\hline HMB & 0.127 & 0.004 & 0.122 & 0.002 & 0.08 \\
\hline \multicolumn{6}{|l|}{ CRP (mg/l) } \\
\hline Placebo & $2 \cdot 0$ & 0.7 & $1 \cdot 1$ & 0.2 & \\
\hline HMB & 1.0 & 0.1 & $1 \cdot 3$ & 0.3 & 0.29 \\
\hline
\end{tabular}

3-MH, 3-methylhistadine; CR, creatinine; CRP, C-reactive protein.

* Probability of treatment difference for the change from 0 to $48 \mathrm{~h}$ post-exercise.

levels were assayed from blood draws before and $48 \mathrm{~h}$ after the resistance exercise protocol. There was a robust increase in serum levels of CK $48 \mathrm{~h}$ post-exercise, indicating the occurrence of muscle damage (Fig. 1). However, the present results demonstrated that an acute bolus ingestion of HMB-FA 30 min before exercise was able to attenuate the rise in CK. Previous studies that assessed muscle damage following a single exercise bout were conducted on untrained individuals who were administered HMB-Ca ${ }^{(16,22)}$. Specifically, van Someren et $a l .{ }^{(22)}$ found that $3 \mathrm{~g} \mathrm{HMB-Ca}$ and $0.3 \mathrm{~g} \alpha$-ketoisocaproate administered daily for 2 weeks before an eccentric elbow flexor bout blunted the rise in $\mathrm{CK}$, and that the supplementation also attenuated declines in one maximal repetition performance compared with placebo supplementation. Moreover, Wilson et al. ${ }^{(16)}$ demonstrated that a single $3 \mathrm{~g}$ serving of HMB-Ca administered $1 \mathrm{~h}$ before exercise in non-resistancetrained males prevented a significant increase in CK, lactate dehydrogenase and quadriceps soreness after an acute bout of exercise. The present study extends this research to a highly trained population, and utilised a more rigorous, multi-joint-centred resistance exercise bout.

Table 3. Effects of $\beta$-hydroxy- $\beta$-methylbutyrate free acid on anabolic and catabolic hormone profile

(Mean values with their standard errors)

\begin{tabular}{|c|c|c|c|c|c|}
\hline & \multicolumn{4}{|c|}{ Time points } & \multirow[b]{3}{*}{$P^{*}$} \\
\hline & \multicolumn{2}{|c|}{0} & \multicolumn{2}{|c|}{$48 \mathrm{~h}$} & \\
\hline & Mean & SEM & Mean & SEM & \\
\hline \multicolumn{6}{|c|}{ Cortisol $(\mu \mathrm{g} / \mathrm{l})$} \\
\hline Placebo & 197 & 12 & 205 & 15 & \\
\hline $\mathrm{HMB}$ & 215 & 14 & 203 & 12 & 0.27 \\
\hline \multicolumn{6}{|c|}{ Free testosterone $(\mathrm{ng} / \mathrm{l})$} \\
\hline Placebo & 1060 & 140 & 1090 & 100 & \\
\hline $\mathrm{HMB}$ & 1090 & 100 & 1040 & 80 & 0.58 \\
\hline \multicolumn{6}{|c|}{ Total testosterone $(\mathrm{ng} / \mathrm{l})$} \\
\hline Placebo & 6140 & 770 & 6290 & 640 & \\
\hline HMB & 7080 & 350 & 7080 & 480 & 0.80 \\
\hline
\end{tabular}

${ }^{*}$ Probability of treatment difference for the change from 0 to $48 \mathrm{~h}$ post-exercise. 


\section{Perceived recovery scale}

Not permitting athletes adequate recovery time is detrimental to obtaining peak performance ${ }^{(23,24)}$. Laurent et al. ${ }^{(25)}$ proposed a 0 (not recovered) to 10 (highly recovered) PRS scale based on the subjective physical and mental feelings of the athlete, as it pertains to their body before a training session. The PRS scale is an effective and valid tool for examining the recovery after a particular training session before a subject commences further training ${ }^{(25)}$. In the present study, subjects noted a significant drop in their PRS $48 \mathrm{~h}$ following the high-volume resistance training session (Fig. 2). However, this decline in perceived recovery was blunted by HMB-FA supplementation relative to placebo. Previous research with HMB had only analysed subjective measures of soreness ${ }^{(16,22)}$. To our knowledge, we are the first to examine the supplement's effect on an athlete's mental perception of their physical readiness to train.

\section{Muscle protein breakdown}

One possible mechanism thought to underlie HMB's capacity to speed recovery is its effects on protein breakdown. Skeletal muscle mass is determined by two competing processes: protein synthesis and protein breakdown. In a catabolic situation, such as acute muscle injury, muscle protein breakdown is increased. As muscle protein is degraded, there is the release of the muscle-specific metabolite, 3-MH, which has been used as an index of myofibrillar protein breakdown ${ }^{(26-28)}$. In the present study, the measure of urinary 3-MH:creatinine ratio approached significance, which may suggest a lower rate of muscle protein breakdown in the HMB-FA supplementation condition, compared with placebo supplementation. Previous research by Nissen et $a l .{ }^{(7)}$ investigated the effects of $\mathrm{HMB}$ during 3 weeks of resistance training. Their results found a decrease in protein breakdown at week 1 , which was decreased even further by the end of week 2 with HMB-Ca supplementation. These findings may indicate a chronic effect of HMB supplementation on protein breakdown. It is important to note that there are non-skeletal muscle sources of 3-MH including gut tissues, which turn over at a more rapid rate than the significantly larger skeletal muscle pool. However, non-skeletal muscle sources do not significantly influence urinary 3-MH output even in extremely catabolic conditions. Furthermore, because the subjects consumed meat-free diets of the same composition and underwent the same workout protocol, it is unlikely that contributions from these other tissues significantly affected the present findings.

\section{Testosterone, cortisol and C-reactive protein}

Previous findings from Kraemer et al. ${ }^{(8)}$ found that chronic supplementation with an HMB-containing supplement resulted in greater increases in resting levels of testosterone and decreases in cortisol levels. Conflicting results exist in untrained populations concerning the role of acute changes in hormones in neuromuscular adaptations ${ }^{(29,30)}$. However, in trained populations similar to those in the present study,
Hakkinen et al. ${ }^{(31,32)}$ and Beaven et al. ${ }^{(33)}$ have demonstrated that resting and acute changes, respectively, in hormone status are correlated with changes in neuromuscular adaptations. In the present study, we examined testosterone (total and free), cortisol and CRP to determine any possible acute effects of HMB-FA on hormone status (anabolic and/or catabolic) and inflammation. The present results demonstrated no significant change in testosterone (free or total), cortisol or CRP levels from pre- to $48 \mathrm{~h}$ post-workout (Tables 2 and 3). Although some researchers have noticed changes in the levels of testosterone and cortisol in blood and saliva after resistance exercise ${ }^{(34-36)}$, samples in those studies were taken either immediately or a few hours after exercise. Changes in hormone status may have occurred at time intervals different from the one chosen for the present study $(48 \mathrm{~h})$. Our postexercise times for sampling were chosen because this is when maximal concentrations of muscle damage markers would be present in the blood. Previous research has indicated that CRP responds to chronic conditions of inflammation ${ }^{(37-39)}$, which may explain why the present results indicated no acute change in the inflammatory marker.

The present study, similar to others, has limitations. Primarily, we did not measure any indices of performance post-supplementation because the present study was designed to measure the acute effects of HMB-FA on muscle damage and recovery. Another limitation concerns our measure of CRP, which may be more indicative of long-term changes in inflammation. Changes in inflammatory markers with greater sensitivity to acute exercise may have yielded more insightful findings. Therefore, we suggest future research examine changes in performance post-supplementation. We also suggest future research sample classes of pro-inflammatory markers such as TNF- $\alpha$, which is more sensitive to acute changes in inflammation ${ }^{(40)}$.

\section{Conclusions}

The present study was the first to investigate the acute effects of an HMB-FA supplement in a resistance-trained population. The present results indicate that acute HMB-FA supplementation administered just before a bout of exercise attenuates serum indices of muscle damage and increases an athlete's mental perception of physical preparedness to train following high-volume, muscle-damaging resistance exercise. These findings suggest that athletes seeking to speed recovery from high-volume, high-intensity training can do so by consumption of HMB-FA 30 min before exercise.

\section{Acknowledgements}

This study was funded through a grant from Metabolic Technologies, Inc. HMB-FA was formulated and supplied by Metabolic Technologies, Inc. The authors' contributions are as follows: J. M. W., R. P. L. and J. M. J. were involved in the study design, training subjects, biochemical analysis of blood and manuscript preparation. J. R., S. M. B. and J. C. F. were involved in the study design, supplement preparation, analysis of 3-MH, data analysis and manuscript preparation. J. A. W.,

in trained populations similar to those in the present study, 
E. M. S. and N. M. D. were critical for data collection, training subjects and data input. J. R. S. and L. E. N. were critical for the study design and manuscript preparation. S. M. C. W. served as the study's sports dietitian and also assisted in the study design. J. M. W., R. P. L., J. M. J., J. A. W., N. M. D., E. M. S. and S. M. C. W. declare that they have no competing interests. J. C. F., S. M. B. and J. R. are employed by Metabolic Technologies, Inc.

\section{References}

1. Renault V, Piron-Hamelin G, Forestier C, et al. (2000) Skeletal muscle regeneration and the mitotic clock. Exp Gerontol 35, 711-719.

2. Jennische E \& Hansson HA (1987) Regenerating skeletal muscle cells express insulin-like growth factor I. Acta Physiol Scand 130, 327-332.

3. Ge Y, Wu AL, Warnes C, et al. (2009) mTOR regulates skeletal muscle regeneration in vivo through kinase-dependent and kinase-independent mechanisms. Am J Physiol Cell Physiol 297, C1434-C1444.

4. Carpinelli RN \& Otto RM (1998) Strength training. Single versus multiple sets. Sports Med [Review] 26, 73-84.

5. Gallagher PM, Carrithers JA, Godard MP, et al. (2000) Betahydroxy-beta-methylbutyrate ingestion, part I: effects on strength and fat free mass. Med Sci Sports Exerc 32, $2109-2115$.

6. Jowko E, Ostaszewski P, Jank M, et al. (2001) Creatine and beta-hydroxy-beta-methylbutyrate (HMB) additively increase lean body mass and muscle strength during a weight-training program. Nutrition 17, 558-566.

7. Nissen S, Sharp R, Ray M, et al. (1996) Effect of leucine metabolite beta-hydroxy-beta-methylbutyrate on muscle metabolism during resistance-exercise training. I Appl Physiol 81, 2095-2104.

8. Kraemer WJ, Hatfield DL, Volek JS, et al. (2009) Effects of amino acids supplement on physiological adaptations to resistance training. Med Sci Sports Exerc 41, 1111-1121.

9. Norton LE \& Layman DK (2006) Leucine regulates translation initiation of protein synthesis in skeletal muscle after exercise. J Nutr 136, 533S-537S.

10. Baxter JH, Carlos JL, Thurmond J, et al. (2005) Dietary toxicity of calcium beta-hydroxy-beta-methyl butyrate (CaHMB). Food Chem Toxicol 43, 1731-1741.

11. Smith HJ, Mukerji P \& Tisdale MJ (2005) Attenuation of proteasome-induced proteolysis in skeletal muscle by \{beta\}hydroxy-\{beta\}-methylbutyrate in cancer-induced muscle loss. Cancer Res 65, 277-283.

12. Smith HJ, Wyke SM \& Tisdale MJ (2004) Mechanism of the attenuation of proteolysis-inducing factor stimulated protein degradation in muscle by beta-hydroxy-beta-methylbutyrate. Cancer Res 64, 8731-8735.

13. Mitch WE \& Goldberg AL (1996) Mechanisms of muscle wasting. The role of the ubiquitin-proteasome pathway. N Eng J Med 335, 1897-1905.

14. Panton LB, Rathmacher JA, Baier S, et al. (2000) Nutritional supplementation of the leucine metabolite beta-hydroxybeta-methylbutyrate (HMB) during resistance training. Nutrition 16, 734-739.

15. Neighbors KL, Ransone JW, Jacobson BH, et al. (2000) Effects of dietary $\beta$-hydroxy- $\beta$-methylbutyrate on body composition in collegiate football players. Med Sci Sports Exerc [Abstract] 32, S60.

16. Wilson JM, Kim JS, Lee SR, et al. (2009) Acute and timing effects of beta-hydroxy-beta-methylbutyrate (HMB) on indirect markers of skeletal muscle damage. Nutr Metab 6, 6.

17. Wilson GJ, Wilson JM \& Manninen AH (2008) Effects of betahydroxy-beta-methylbutyrate (HMB) on exercise performance and body composition across varying levels of age, sex, and training experience: a review. Nutr Metab (Lond) 5,1 .

18. Fuller JC Jr, Sharp RL, Angus HF, et al. (2011) Free acid gel form of beta-hydroxy-beta-methylbutyrate (HMB) improves HMB clearance from plasma in human subjects compared with the calcium HMB salt. BrJ Nutr 105, 367-372.

19. Gilbert G \& Lees A (2005) Changes in the force development characteristics of muscle following repeated maximum force and power exercise. Ergonomics 48, 1576-1584.

20. Rathmacher JA, Link GA, Flakoll PJ, et al. (1992) Gas chromatographic/mass spectrometric analysis of stable isotopes of 3-methylhistidine in biological fluids: application to plasma kinetics in vivo. Biol Mass Spectrom 21, 560-566.

21. Cohen J (1988) Statistical Power Analysis for the Behavioral Sciences, 2nd ed. Hillsdale, NJ: L. Erlbaum Associates.

22. van Someren KA, Edwards AJ \& Howatson G (2005) Supplementation with beta-hydroxy-beta-methylbutyrate (HMB) and alpha-ketoisocaproic acid (KIC) reduces signs and symptoms of exercise-induced muscle damage in man. Int J Sport Nutr Exerc Metab 15, 413-424.

23. Kentta G \& Hassmen P (1998) Overtraining and recovery. A conceptual model. Sports Med [Review] 26, 1-16.

24. Laursen PB \& Jenkins DG (2002) The scientific basis for high-intensity interval training: optimising training programmes and maximising performance in highly trained endurance athletes. Sports Med [Review] 32, 53-73.

25. Laurent CM, Green JM, Bishop PA, et al. (2011) A practical approach to monitoring recovery: development of a perceived recovery status scale. J Strength Cond Res 25 620-628.

26. Young VR \& Munro HN (1978) Ntau-methylhistidine (3-methylhistidine) and muscle protein turnover: an overview. Fed Proc 37, 2291-2300.

27. Munro HN \& Young VR (1978) Urinary excretion of $\mathrm{N}$-gamma-methylihistidine (3-methylihistidine): a tool to study metabolic responses in relation to nutrient and hormonal status in health and disease of man. Am J Clin Nutr 31, 1608-1614.

28. Elia M, Carter A, Bacon S, et al. (1981) Clinical usefulness of urinary 3-methylhistidine excretion in indicating muscle protein breakdown. Br Med J 282, 351-354.

29. West DW, Burd NA, Tang JE, et al. (2010) Elevations in ostensibly anabolic hormones with resistance exercise enhance neither training-induced muscle hypertrophy nor strength of the elbow flexors. J Appl Physiol 108, 60-67.

30. Ronnestad BR, Nygaard H \& Raastad T (2011) Physiological elevation of endogenous hormones results in superior strength training adaptation. Eur J Appl Physiol 111, 2249-2259.

31. Hakkinen K, Pakarinen A, Alen M, et al. (1988) Neuromuscular and hormonal adaptations in athletes to strength training in two years. $J$ Appl Physiol 65, 2406-2412.

32. Hakkinen K, Pakarinen A, Alen M, et al. (1985) Serum hormones during prolonged training of neuromuscular performance. Eur J Appl Physiol Occup Physiol 53, 287-293.

33. Beaven CM, Cook CJ \& Gill ND (2008) Significant strength gains observed in rugby players after specific resistance exercise protocols based on individual salivary testosterone responses. J Strength Cond Res 22, 419-425.

34. Nunes JA, Crewther BT, Ugrinowitsch C, et al. (2011) Salivary hormone and immune responses to three resistance exercise 
schemes in elite female athletes. J Strength Cond Res 25 , 2322-2327.

35. Rogers RS, Dawson AW, Wang Z, et al. (2011) Acute response of plasma markers of bone turnover to a single bout of resistance training or plyometrics. J Appl Physiol 111, 1353-1360.

36. Szivak TK, Hooper DR, Kupchak BK, et al. (2012) Adrenal cortical responses to high intensity, short rest, resistance exercise in men and women. J Strength Cond Res (Epublication ahead of print version 3 May 2012).

37. Milias GA, Nomikos T, Fragopoulou E, et al. (2005) Effects of eccentric exercise-induced muscle injury on blood levels of platelet activating factor (PAF) and other inflammatory markers. Eur J Appl Physiol 95, 504-513.

38. Malm C, Sjodin TL, Sjoberg B, et al. (2004) Leukocytes, cytokines, growth factors and hormones in human skeletal muscle and blood after uphill or downhill running. J Physiol 556, 983-1000.

39. Goto K, Takahashi K, Yamamoto M, et al. (2008) Hormone and recovery responses to resistance exercise with slow movement. J Physiol Sci 58, 7-14.

40. Malm C \& Yu JG (2012) Exercise-induced muscle damage and inflammation: re-evaluation by proteomics. Histochem Cell Biol 138, 89-99. 